\title{
Development of Empirical formula Prediction on Critical Impact Energy for Perforation Phenomena on Concrete Structures
}

\author{
Qadir Bux alias Imran Latif \\ Faculty of Civil and Environmental Engineering, Universiti Tun Hussein Onn Malaysia (UTHM) \\ 86400, Parit Raja, Johor, Malaysia \\ Tel: 60-1-9701-3878 E-mail: imranqazi37@gmail.com \\ Ismail Abdul Rahman \\ Faculty of Civil and Environmental Engineering, Universiti Tun Hussein Onn Malaysia (UTHM) \\ 86400, Parit Raja, Johor, Malaysia
}

Tel: 60-1-2787-6360 E-mail: ismailar@uthm.edu.my

Ahmad Mujahid Ahmad Zaidi (Corresponding author)

Department of Mechanical Engineering, Faculty of Engineering, National Defence University (UPNM)

Kem Sungai Besi, 57000 Kuala Lumpur, Malaysia

Tel: 60-1-7478-3139 E-mail: mujahid@upnm.edu.my

\begin{abstract}
Concrete is basic construction material used for most structures. However, the vital structures have to be designed as selfprotective such as nuclear plants, power plants, weapon industries, weapons storage places, water retaining structures, highway structures, and etc. These structures should have to be offer self shield against any natural disaster tragedy incidents and intentionally produced horrible incidents such as dynamic loading, incident occurs in nuclear plants, terrorist attack, missile attack, tsunami and etc. In modern science, the impact energies are crucial way to study the local impact effects on concrete structures together with the influence of relative target thickness $(\mathrm{H} / \mathrm{d})$. This study is focused on minimum required kinetic energy for perforation of the concrete structures generated by flat nose hard missile in terms of relative target thickness $(\mathrm{H} / \mathrm{d})$, by using curve fitting empirical study with the implementation of dimensional analysis of non-dimensional numbers. For verification, the proposed developed empirical formula was well compared with other established empirical formulae such as NDRC, UMIST and Semi Empirical formulae and it is expected that the outcome of the proposed formulae can be applied in design recommendations and design procedures for determining the dynamic reaction of the concrete target to foil perforation of flat nose hard missile.
\end{abstract}

Keywords: Perforation, Concrete, Critical, Kinetic energy, Empirical, Flat nose, Hard missile

\section{Introduction}

Over the years, concrete is very commonly used construction material for the defensive and civil applications to protect structures from local and explosive impact loads. The effects of the local impact of hard missile on concrete structures have been studied since the mid of 17 th century because of continuous military interest in designing high performance missiles and high performance protective barriers. Projectile exists in a long range with variation in sizes, shapes, velocity, weight, density, hardness, such as bullets, fragments, tornado, bomb, missile etc. The projectile may be classified as 'Hard' and 'Soft' depending upon deformability of projectile with respect to target's deformation (Kennedy 1976), (Q. M. Li et al 2005), and (P. Koechlin and S. Potapov 2009). Deformation of hard missile is considerable smaller or negligible compared with target's deformation. Almost in all cases hard missiles are considered as non - deformable or rigid. However, 'Soft' missile deforms itself considerably well as compared to target's deformation (Kennedy 1976), (Q. M. Li et al 2005), and (P. Koechlin and S. Potapov 2009).

In general, the local impact effect of hard missile on concrete structures can be studied in three ways, (i). Empirical Studies (predict empirical formula based on experimental data), (ii). Analytical Studies (create formula based on physical laws and compared with experimental data), and (iii), Numerical Simulation (based on computer based material model generate results and compared with experimental data). Empirical formulae based on experimental data are especially important in this field due to the complexity of the local impact phenomena. Some of these have been summarized in previous review papers. However, particular empirical formulae for perforation limits are often formulated by curve-fitting test data and most of them are unit-dependent and limited by the range of the validity, and only being applicable strictly within the limits 
of the tests from which the data were acquired (Kennedy 1976 and Q. M. Li et al 2005). Critical impact kinetic energy is essential technique to determine the local impact effects. The way in which the kinetic energy is distributed through the concrete target is also noteworthy in determining its response against local impact effects (perforation). Literature review showed that fine-looking work have been done on predictions of perforation. This study shows the influence of relative target thickness $(\mathrm{H} / \mathrm{d})$ on the required critical impact kinetic energy which may cause the complete or just perforation in concrete targets, when it is subjected to the impact of flat nose hard missile. This study is based on empirical formula predicted by using curve fitting data method by polynomial equation.

\section{Literature Review}

The effects of the local impact of hard missile on concrete structures have been studied since the mid of 17 th century because of continuous military interest in designing of high performance missiles and high performance protective barriers (Q.M. Li et al 2005). A review of research work exposed that peak studies about concrete structures against dynamic loading were conducted from the early 1940s (Z. Liang, et al 2007). However, most of the research work ceased shortly after World War - II and was not resumed until 1960s (Z. Liang, et al 2007). The intensive study on concrete targets against local impact effects of hard missiles in the nuclear industry re-initiated since five decades ago. Kennedy (1976) provided an early review of the concrete response against local impact effects of hard missile for nuclear industries and recommended impact force time history theory (Kennedy 1976 and Q. M. Li et al 2005). Various empirical studies were conducted to explore local impact effects of hard missile on concrete structures, a review of these studies were discussed intensively in previous publications, e.g., Kennedy (1976), Bangash (1993), Williams (1994), Corbett et al. (1996), and Q.M Li (2005).

The extensive empirical studies were conducted in past for predicting the perforation limit, the most used empirical formulae for designing of concrete structures to overcome perforation of hard missile are Modified Petry formula, Ballistic Research Laboratory (BRL) formula, Army corps of engineers (ACE) formula, Modified NDRC formula (Q. M. Li et al 2005). Other than these Degen perforation formula, Hughes formula based on tensile strength of concrete, also can be used to predict perforation limit for concrete targets (Q. M. Li et al 2005). It is noted that all above mentioned empirical formulae used to predict perforation limits are dependent of penetration depth. Furthermore, UKAEA formula based on Barr's assumptions for ballistic limit, CEA - EDF perforation formula for reinforced concrete, and Criepi formula are used to predict perforation limit of concrete (Q. M. Li et al 2005).

Critical impact kinetic energy is essential technique to determine the local impact effects. It is essential to determine the effects of kinetic energy and relative target thickness on local impact effect with dimensional analysis of non-dimensional numbers (Q. M. Li et al 2006). The critical impact kinetic energy, which can produce perforation in to the concrete target with the impact of hard missile, can be calculated by using empirical formula derived by UMIST (Q. M. Li et al 2005). Furthermore, Q.M. Li et al (2006) modified the UMIST, NDRC, and Semi empirical formula for required critical impact energy to perforate concrete targets impacted with flat nose hard missile in terms of dimensional analysis of non - dimensional numbers (Q. M. Li et al 2006). However, literature recommends that the NDRC formula predicts more accurate results among all other above mentioned formulae (Kennedy 1976 and Q. M. Li et al 2005).

\section{Local Impact Effects}

Local impact effect is briefly sub-divided in below explained processes:

\subsection{Radial Cracking}

When projectile colloids with concrete target with certain velocity, it results radial cracks originated from the point of impact within the target in every direction (Q. M. Li et al 2005).

\subsection{Spalling}

The ejection of material of target from front face (impacted face) due to impact of hard projectile is called spalling. Spalling produces spall crater in the surrounding area of impact. Spall crater is the total damaged portion of peeling off material from target on impacted face (Kennedy 1976 and Q. M. Li et al 2005).

\subsection{Penetration}

Penetration is defined as the digging of missile into the target body afar from the thickness of spall crater. The lengthwise measurement of dig is called penetration depth (Kennedy 1976 and Q. M. Li et al 2005).

\subsection{Cone cracking and Plugging}

During penetration missile colloids with rear border of target and generates curved shear cracks in the shape of bell plug is called cone cracking. And than missile continues penetrating through target, it forces plug and shears-off the surrounding material of target is called plugging. This process generates rapid change into the behavior of target (Q. M. Li et al 2005).

\subsection{Scabbing}

Ejection of target material from back face of target is called scabbing (Kennedy 1976 and Q. M. Li et al 2005). 


\subsection{Perforation}

Perforation means complete passage or complete crossing of projectile through the target. It causes missile to extend penetration hole through scabbing crater and exit from the rear face of target (Kennedy 1976 and Q. M. Li et al 2005).

\section{$<$ Figure $1>$}

\section{Critical Impact Energies and Non-dimensional Number Analysis}

It is important to consider the mechanics of the impact phenomena and thus deduce the relevant non-dimensional numbers that could be involved in perforation limit analyses (Q. M. Li et al 2006). When a non-deformable, flat-nosed projectile strikes with a concrete target, perforation could occur due to the shear failure (Q. M. Li et al 2003 and 2006). The perforation shear failure can be occur in concrete when the total penetration resistance in front of the projectile nose equals to the shear resisting force offered by the remaining thickness of the target, which initiates plug formation and the shear-out of the plug of the rear face of concrete target (Q. M. Li et al 2003). The shear stress $\tau_{f}$ is clearly major reason involved in perforation of concrete target. Therefore, the perforation limit, i.e., the minimum target thickness to prevent perforation, is generally defined by:

$$
e=G\left(M, V_{o}, d, \rho, f_{c}, f_{t}, E, \tau_{f}\right)
$$

Where $\rho, \mathrm{E}$ and $\mathrm{fc}$ and $\mathrm{ft}$ are the density, Young's modulus and unconfined compressive and tensile strengths (stresses) of the concrete target, respectively. $\mathrm{M}$ and $V_{o}$ are the mass and the initial impact velocity of a projectile and $\mathrm{d}$ is the (cylindrical) projectile shank diameter, and $\tau_{f}$ is the shear strength of concrete. A non-dimensional analysis for perforation of concrete targets based on Equation (1) leads to:

$$
e / d=G\left(E_{k} / f_{c} d^{3}, M / \rho d^{3}, f_{t} / f_{c}, E / f_{c}, \tau_{f} / f_{c}\right)
$$

Where $E_{k}=(1 / 2) M V_{o}^{2}$ is the kinetic energy of the missile. Although the perforation is normally selected as a design parameter in empirical formulae, the critical impact velocity to cause perforation for given target thickness is another important parameter associated with perforation(Q. M. Li et al 2006). The required critical impact energy for perforation can be expressed by:

$$
E_{c p} / f_{c} d^{3}=G\left(M / \rho d^{3}, H / d, f_{t} / f_{c}, E / f_{c}, \tau_{f} / f_{c}\right)
$$

Where $\mathrm{H}$ is the thickness of the concrete target and for a given target, $f_{t} / f_{c}$ and $E / f_{c}$ may be considered as constants.

\subsection{Development of New Empirical Formula}

Empirical formulae based on experimental data are especially important in this field due to the complexity of the phenomena. These empirical formulae, e.g., for penetration depth, the scabbing and perforation limits, are often formulated by curve fitting test data, and thus, their validity is only guaranteed in their valid application range.

The new empirical formula for calculation of required critical energies for perforation on concrete targets based on $\mathrm{H} / \mathrm{d}$ is developed by using the polynomial equations. The equation for the derivation of formula is equation of $3^{r d}$ order polynomial equation.

The general form of basic polynomial equation is:

$$
\left.y=a x^{n}+b x^{n-1}+.+c x+d\right)
$$

Based on above general equation, equation (4) the polynomial equation of $3^{\text {rd }}$ degree is:

$$
\left.y=a x^{3}+b x^{2}+c x+d\right)
$$

Based on equation (5) the general equation for required energies to perforate the concrete target is:

$$
E_{c p} / f_{c} d^{3}=a(H / d)^{3}+b(H / d)^{2}+c(H / d)+d
$$

Where $E_{c p}$ is required critical impact kinetic energy of missile for perforation of concrete target, $f_{c}$ is unconfined compressive stress of concrete, $\mathrm{d}$ is (cylindrical) projectile shank diameter, and $\mathrm{H}$ is total thickness of concrete target.

Based on equation (6) the critical impact kinetic energy required for perforation of concrete target is: 


$$
E_{c p} / f_{c} d^{3}=0.174(H / d)^{3}+0.169(H / d)^{2}+0.0577(H / d)+0.2969 \text { for }(0.69 \leq H / d \leq 14.86)
$$

The above non - dimensional equation (7), can be used within there applicable given range depend on $\mathrm{H} / \mathrm{d}$.

\section{Result and Discussion}

The proposed developed formula examined for the prediction of minimum required critical kinetic energy of flat nose hard missile to perforate concrete targets. Results obtained from proposed developed formula is relatively closer as compared to the experimental results.

The predictions based on four formulae have been compared with the experimental data. It is found that the UMIST formula predicts the lowest values of the required critical impact energy for perforation in comparison with other three groups of empirical formulae. The predicted results are consistently lower than the experimental results. Although overall predictions based on both NDRC and semi-empirical formulae are closer to experimental data than those based on UMIST formulae, the NDRC and semi-empirical formulae sometimes overestimate the required critical impact kinetic energy for the perforation of concrete target.

Among all these four formulae the proposed developed formula gives relatively average results as compared to other formulae. Like NDRC and semi-empirical formulae the proposed developed formula also sometimes gives under prediction results and also sometimes over prediction values.

Most empirical formulae for the prediction of local impact effects of hard missile on concrete targets have their specific range of parameters, and the validity of those empirical formulae are only guaranteed in their valid application range. Like other empirical formulae, proposed formula also can be used within the range of $0.69 \leq H / d \leq 14.86,24.15 \leq f_{c} \leq$ $50.20(\mathrm{MPa}), 17.50 \leq d \leq 305.00(\mathrm{~mm}), 0.92 \leq M \leq 309.00(\mathrm{~kg}), 28.98 \leq V_{o} \leq 427.00(\mathrm{~m} / \mathrm{sec})$, for normal impact caused by flat nose hard missile on concrete targets.

$$
<\text { Figure } 2>
$$

\section{Conclusion}

The influence of the relative target thickness $(\mathrm{H} / \mathrm{d})$ on the critical impact energies, at which perforation occurs in concrete targets may be initiated by hard missile, has been investigated in this paper. A proposed empirical formula for required critical impact kinetic energies for perforation of concrete target has been developed. The results obtained from proposed formula are also compared with other empirical formulae likes of UMIST, NDRC and Semi Empirical formula. Since UMIST formulae have been verified by a large collection of experimental data conducted by nuclear industries, and NDRC formulae have a long history and wide range of applications, literature recommended NDRC formula as the most appropriate formulae for the preliminary impact design of concrete targets in nuclear facilities. However the results obtained from proposed developed formula shows relatively average predictions, as compared to other formulae, and sometimes under predict and over predict results. Therefore, appropriate safety factor should be considered for the application of NDRC, semi-empirical and newly developed formula for perforation.

\section{References}

Bangash MYH. (1993). Impact and explosion: structural analysis and design. Boca Raton, FL: CRC Press; 1993. Williams MS. Modeling of local impact effects on plain and reinforced concrete. ACI Struct J, 91 (2):178-87.

Corbett GG, Reid SR, Johnson W. (1996). Impact loading of plates and shells by free-flying projectiles: a review. Int $J$ Impact Eng, 18:141-230.

Kennedy RP. (1976). A review of procedures for the analysis and design of concrete structures to resist missile impact effects. Nucl. Eng. Des, 37:183-203.

Li QM, eid SR, Ahmad-Zaidi AM. (2006). Critical impact energies for scabbing and perforation of concrete target. Nucl Eng Des, 236:1140-8.

Li QM, Reid SR, Wen HM, Telford AR. (2005). Local impact effects of hard missiles on concrete targets. Int J Impact Eng, 32 (1-4): 224-84.

Li QM, Tong DJ. (2003). Perforation thickness and ballistic performance of concrete target subjected to rigid projectile impact. ASCE J Eng Mech, 129(9):1083-91.

Pierre Koechlin, Serguei Potapov. (2009). Classification of soft and hard impacts - Application to aircraft crash. Nucl. Eng. Des, 239: 613-618.

Zhi-Liang Wang, Yong-chi Li, R.F Shen, J.G. Wang. (2007). Numerical study on craters and penetration of concrete slab by ogive nose steel projectile. Computers and Geotechnics, 36 : 1-9. 


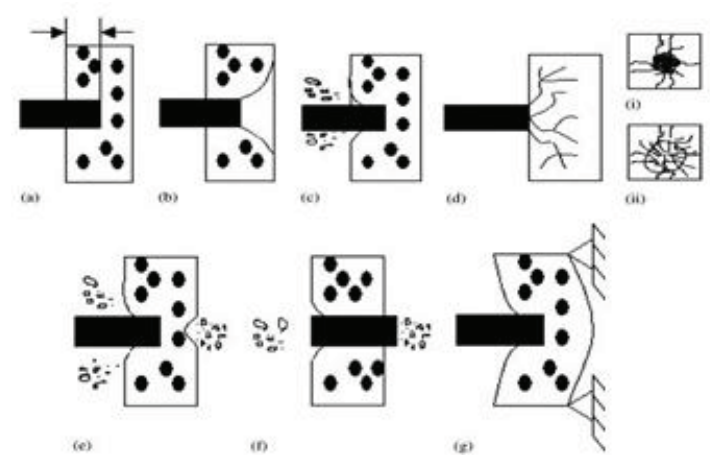

Figure 1. Explains the local impact phenomena caused by hard projectile. (a)Penetration, (b) Cone cracking and Plugging, (c) Spalling, (d)Radial cracking, (e) Scabbing, (f) Perforation, and (g) Global phenomena (Q. M. Li et al 2005).

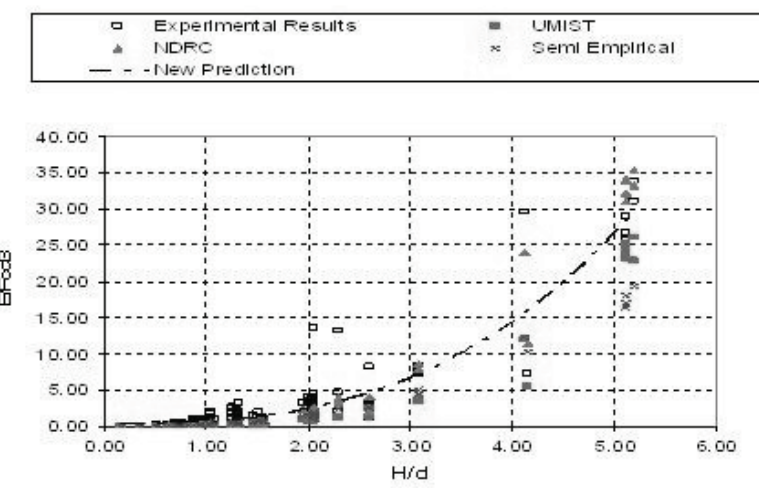

Figure 2. Explains the results of newly developed formula and comparison with prediction of other formulae

\section{Nomenclature}

$\mathrm{d} \rightarrow$ cylindrical projectile shank diameter.

$\mathrm{E} \rightarrow$ Young's Modulus of Elasticity.

$\mathrm{Ek} \rightarrow$ Impact kinetic energy of the projectile.

$\mathrm{Ec} \rightarrow$ Critical impact energy of projectile.

Ecp $\rightarrow$ Critical impact energy of the projectile for perforation.

$\mathrm{fc} \rightarrow$ uni-axial compressive strength of concrete target.

$\mathrm{ft} \rightarrow$ uni-axial tensile strength of concrete target.

$\mathrm{H} \rightarrow$ Concrete target thickness.

$\mathrm{M} \rightarrow$ Mass of the projectile.

$\mathrm{Vc} \rightarrow$ Critical impact velocity of projectile for the occurrence of perforation.

Vo $\rightarrow$ Impact velocity of projectile.

$\mathrm{e} \rightarrow$ Perforation limit.

$\rho \rightarrow$ Density of concrete target. 